\title{
APLICAÇÃO DO MÉTODO HIBRIDO AHP-TOPSIS-2N PARA ESCOLHA DE SISTEMA DE TRANSPORTE URBANO: UM ESTUDO DE CASO NO MUNICÍPIO DE MAGÉ/RJ
}

\author{
Ana Lívia Caitano (Universidade do Grande Rio) livia.alves.caitano@gmail.com \\ Marcos dos Santos (IME) marcosdossantos_doutorado_uff@yahoo.com.br \\ Paulo Afonso Lopes da Silva (IME) pauloafonsolopes@uol.com.br \\ Daniel Augusto de Moura Pereira (UFCG) danielmoura@ufcg.edu.br \\ Igor Pinheiro de Araújo Costa(UFF) costa_igor@id.uff.br
}

\begin{abstract}
Resumo
O deslocamento no espaço é um pré-requisito para o atendimento das necessidades econômicas e sociais da população. A ineficiência do transporte urbano impacta principalmente as famílias de renda mais baixa. Os efeitos negativos vão bem além do desconforto e perda de qualidade de vida: quanto maior o tempo (e custos associados) de deslocamento, menos disponibilidade de tempo para educação e menor participação na força do trabalho. Para chegar à capital, o morador de Magé possui algumas alternativas de transporte (ônibus, carro particular, integração ônibus-trem, van) que possuem inúmeras variáveis tais como: tempo de viagem, acessibilidade, custo, conforto, segurança. Tornando essa escolha uma tarefa complexa. Diante disto, foi utilizado o Método AHP-TOPSIS-2N para seleção de um meio de transporte, para a chegada do cidadão mageense ao centro do Rio de Janeiro, chegando a conclusão de que o modal do tipo ferroviário seria a melhor opção para o caso estudado, tendo em vista o baixo custo reduzido e tempo de deslocamento.
\end{abstract}

Palavras-Chaves: Transporte público; Método Multicritério; AHP-TOPSIS-2n

\section{Introdução}

O deslocamento no espaço é um pré-requisito para o atendimento das necessidades econômicas e sociais da população. Segundo estudo da ONU, "a mobilidade urbana é essencial para o desenvolvimento social e econômico e permite às pessoas ter acesso a serviços, oportunidades de trabalho, de educação, de relações sociais e de desfrutar plenamente da cidade" (ONU, 2012).

No Brasil, principalmente nos grandes centros urbanos, como o estado do Rio de Janeiro, a ausência de um planejamento urbano eficiente representa um dos maiores problemas do Estado (assunto de pauta em todas as eleições políticas). Dados da pesquisa feita em 2015 pela Federação das Indústrias do Estado do Rio de Janeiro (FIRJAN), evidenciam que os prejuízos ao estado alcançam a marca R\$ 19 bilhões (cerca de 6\% do PIB). (FIRJAN, 2015).

A ineficiência do transporte urbano impacta principalmente as famílias de renda mais baixa. Os efeitos perversos vão bem além do desconforto e perda de qualidade de vida: quanto maior o tempo (e custos associados) de deslocamento, menos disponibilidade de tempo para educação e menor participação na força do trabalho. Cria-se um ciclo vicioso: o indivíduo de baixa qualificação tem rendimento menor e, por isso, mora mais longe, gastando mais tempo no deslocamento, tendo menos tempo para participar de atividades de educação ou qualificação que poderiam elevar sua remuneração. Dessa forma, as deficiências no sistema 
de transporte público transformam-se em mecanismo de exclusão social, principalmente na periferia das regiões metropolitanas. (Young; Aguiar \& Possas, 2012).

Segundo estudo da Firjan que analisou os deslocamentos dos moradores de 19 municípios do estado do Rio de Janeiro, o município de Magé ocupou a $4^{\circ}$ colocação, registrando uma média de 162 minutos gastos para o deslocamento do trabalhador no trajeto casa-trabalho-casa, resultando em prejuízos de cerca de 6\% do PIB metropolitano (FIRJAN, 2015).

Neste sentido, para chegar à capital do Estado do Rio de Janeiro, o morador de Magé possui algumas alternativas de transporte, tais como ônibus, carro particular, integração ônibus-trem e van, cada uma delas com suas características de tempo de viagem, acessibilidade, custo, conforto, segurança, tornando essa escolha, muitas vezes, uma tarefa complexa.

A metodologia multicritério de apoio à decisão se trata de uma ferramenta que permite construir conhecimento do contexto de um problema com múltiplas alternativas, hierarquizando-as de acordo com critérios estabelecidos, auxiliando o decisor para a tomada de decisão. Portanto, este trabalho tem como objetivo indicar qual a melhor alternativa de deslocamento do morador de Magé/RJ até a Capital daquele Estado com auxílio da metodologia multicritério AHP-TOPSIS 2N.

\section{Fundamentação Teórica}

\subsection{Transporte Urbano}

A mobilidade urbana pode ser interpretada como "a capacidade dos indivíduos se moverem de um lugar para outro dentro das cidades" (Cardoso, 2018). Está relacionada com os deslocamentos diários (viagens) de determinada população no espaço urbano - não apenas sua efetiva ocorrência, mas também a possibilidade ou facilidade de ocorrência destas viagens (Cardoso, 2018).

Para Silva et al. (2006), o transporte urbano compõe o conjunto de serviços públicos (água, luz, esgoto, etc.) essenciais para o desenvolvimento das cidades, destacando-se dentre os demais. Segundo os autores, o termo transporte urbano é empregado para designar o deslocamento de pessoas e produtos realizados no interior das cidades.

O sistema de transporte exerce o papel de ligação entre as pessoas e os equipamentos urbanos, destacando a importância em garantir a equidade do uso do espaço urbano. Considerando o caráter social do transporte, sua disponibilidade deve ser garantida pelo estado, caracterizando-o como um serviço público (Tyler, 2002).

Se por um lado eles são os facilitares essenciais do desenvolvimento econômico, pois possibilitam o transporte de pessoas e mercadorias dentro das cidades, por outro, contribuem significativamente para a formação de congestionamentos, acidentes, transformações climáticas, problemas de saúde que surgem devido à poluição atmosférica e sonora, e relacionados aos automóveis, contribuem para o aumento da desigualdade social.

Apesar desse cenário relativamente adverso, para Duarte (2012), é possível observar também ao longo dos últimos anos o desenvolvimento maior de tecnologias e formas de se transportar pessoas em massa com qualidade, segurança, conforto e eficácia, de maneira a se adequarem a realidade econômica local, com reduzidos valores de investimento inicial, prospectando períodos de tempo para a sua implantação cada vez menores. A esse contexto podem ser 
citados como soluções mais difundidas atualmente no contexto brasileiro o Bus Rapid Transit (BRT), os ônibus tradicionais (com corredores exclusivos), o Veículo Leve sob Trilhos (VLT) e o metrô (IPEA, 2011; Miranda e Silva, 2012).

Mesmo com os avanços atuais, estas tecnologias ainda são inacessíveis a maioria das regiões mais afastadas da metrópole, segundo estudo revelado pela Associação Nacional de Transporte Urbano (NTU) os ônibus e demais transporte coletivos urbanos rodoviários são responsáveis pelo deslocamento de cerca de 355 milhões de passageiros por mês.

Contudo, a falta de infraestrutura para o transporte coletivo, com redes insuficientes de corredores e faixas, é um dos motivos apontados pela entidade para o atual quadro: o ônibus cada vez mais lentos e menos atrativos. Ainda segundo o estudo, no Estado do Rio de Janeiro, entre 2013 e 2017 a queda da demanda de passageiros a utilizarem os sistemas de ônibus diminui cerca de $25,9 \%$.

\subsection{Modelo Multicritério de Apoio à Decisão}

A Pesquisa Operacional é uma ferramenta matemática frequentemente aplicada no apoio a tomada de decisão, em situações reais ou em um problema no ambiente de negócios (Barbosa e Zanardini, 2015). Santos et al, (2016) afirmam que a Pesquisa Operacional atua em cinco grandes áreas que se inter-relacionam, conforme apresentado na Figura 1.

Figura 1 - Áreas da Pesquisa Operacional

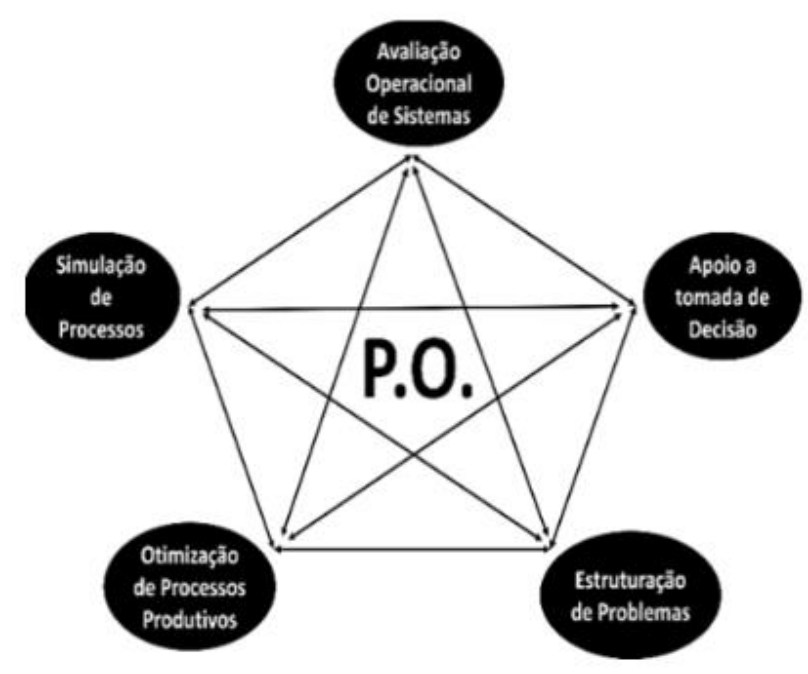

Fonte: Santos et. al. (2016)

Os modelos multicritério têm como principal objetivo tomar as decisões baseadas na descrição dos critérios, em que cada um tem suas alternativas com seus respectivos pesos. Esses pesos definirão qual a decisão mais satisfatória a ser tomada. Quando as decisões são tomadas em grupos os pesos ou preferências individuais são combinados para formar uma decisão conjunta do grupo (Gomes, 2012). Assim, as alternativas indicadas são colocadas perante as partes interessadas, as diferentes possibilidades e respectivos critérios, viabilizam escolha da decisão mais satisfatória, sendo que os agentes envolvidos podem interferir no processo decisório. Segundo Caitano et al. (2019), quando há divergências entre os decisores, estas metodologias formam uma base para discussões, permitindo uma melhor percepção do problema. 
Os Métodos de Apoio Multicritério à Decisão podem ser classificados de diversas formas, a mais comum é dividi-los entre métodos da Escola Francesa e métodos da Escola Americana (Mendonça e Rangel, 2017). Tal qual Bella e Costa (2016), é referenciado na literatura internacional como MCDA (Multicriteria Decision Aid) o estudo de análise multicritério ou auxilio multicritério está fundamentado na utilização de métodos multicritério para solução de questões de seleção, ranqueamento e sobreclassificação.

De acordo com Vilas Boas (2005), as abordagens multicritério se constituem em formas de modelar os processos de decisão, onde entram em jogo: uma decisão a ser tomada, eventos desconhecidos que podem afetar os resultados, possíveis cursos de ação além do próprio resultado, funcionando assim, como base para discussão, principalmente nos casos onde há conflitos entre os decisores, ou ainda quando a percepção do problema pelos vários agentes envolvidos ainda não está totalmente consolidada.

Para os problemas de decisão que possuem como enfoque um conjunto de dados e variáveis discretas, existem diversas técnicas capazes de suprir tal demanda, podendo estas ser separadas, segundo os pressupostos de Greco et al. (2012) e Gomes e Gomes (2012) em três grupos distintos: a Escola Americana, Francesa e a Mista, onde a primeira determinada conforme o paradigma racionalista, relacionado a realização dos julgamentos das diversas etapas da estrutura hierárquica até se chegar a uma solução ótima ao problema e as condições do estado da arte proposto, gerando assim para cada critério funções utilidade capazes de exprimir o seu comportamento em analogia as alternativas, sendo alguns de seus modelos a Multi-attribute Utility Theory (MAUT), o Analytic Hierarchical Process (AHP), o Analytic Network Process (ANP), o Simple Multi-Attribute Rating Technique (SMART) e o Simple Multi-Attribute Rating Technique Extended to Ranking (SMARTER) (Figueira et al., 2005; Gomes e Gomes, 2012).

\subsection{O método AHP-TOPSIS-2N}

O método AHP-TOPSIS-2N é um híbrido entre o Método de Análise Hierárquica (AHP) e o Método Technique for Order Preferences by Similarity to Ideal Solution (TOPSIS).

O método AHP foi originalmente proposto por Thomas L. Saaty na década de 1970 e se consolidou na área de multicritério como o método mais utilizado na atualidade (Russo e Camanho, 2015).

O Decision Support Systems Glossary (DSS, 2006) define AHP como: "uma aproximação para tomada de decisão que envolve estruturação de multicritérios de escolha numa hierarquia. $\mathrm{O}$ método avalia a importância relativa desses critérios, compara alternativas para cada critério, e determina um ranking total das alternativas".

Segundo Costa (2014), o método AHP consiste basicamente nas seguintes etapas: Definir o objetivo a ser alcançado; definir as alternativas; definir uma hierarquia de critérios mais facilmente analisáveis e comparáveis; avaliar as alternativas duas a duas em relação aos critérios; e determinar a avaliação global de cada alternativa e aplicar os cálculos demonstrados na Figura 2. 
Figura 2 - Resumo do Método AHP

\begin{tabular}{|c|c|c|c|}
\hline \multicolumn{4}{|c|}{ Resumo do Método AHP segundo Saaty } \\
\hline Ordem & $\mathrm{N}^{2} \mathrm{Eq}$. & Equação & Descrição da Equação \\
\hline 19 Passo & Eq. 1 & $\left|\begin{array}{ccccc}\mathrm{a} 11 & \mathrm{a} 12 & \mathrm{a} 13 & \ldots & \mathrm{a} 1 \mathrm{n} \\
\mathrm{a} 21 & \mathrm{a} 22 & \mathrm{a} 23 & \ldots & \mathrm{a} 2 \mathrm{n} \\
\cdot & \cdot & \cdot & \ldots & \cdot \\
\dot{*} & \cdot & \cdot & \ldots & \cdot \\
\text { an1 } & \text { an2 } & \text { an3 } & \ldots & . \\
\text { and }\end{array}\right|$ & $\begin{array}{l}\text { Formação das matrizes de decisão. Expressa o } \\
\text { número de vezes em que uma alternativa } \\
\text { domina ou é dominada pelas demais (ARAYA, } \\
\text { CARIGNANO e GOMES, 2004). }\end{array}$ \\
\hline 2 - Passo & Eq. 2 & $w_{i}=\left(\prod_{i=1} w_{i j}\right)$ & $\begin{array}{l}\text { Cálculo do Autovetor (Wi). Consiste em } \\
\text { ordenar as prioridades ou hierarquias das } \\
\text { caracteristicas estudadas (COSTA, 2006). }\end{array}$ \\
\hline 3 P Passo & Eq. 3 & $\mathrm{~T}=\left|\frac{W_{1}}{\Sigma W_{1}}, \frac{W_{2}}{\Sigma W_{i}}, \frac{W_{3}}{\Sigma W_{1}}\right|$ & $\begin{array}{l}\text { Cálculo de Normalização dos autovetores } \\
\text { possibilita a comparabilidade entre os critérios } \\
\text { e alternativas (COSTA, 2006). }\end{array}$ \\
\hline 49 Passo & Eq. 4 & $\lambda_{\text {máx }}=\mathrm{T} \times \mathrm{W}$ & $\begin{array}{l}\text { Indice que relaciona os critérios da Matriz de } \\
\text { Consistência e os pesos dos critérios (COSTA, } \\
\text { 2006). }\end{array}$ \\
\hline 5 Passo & Eq. 5 & $I C=\frac{\lambda \text { máx. }-n}{(n-1)}$ & $\begin{array}{l}\text { Índice de Consistência (IC). Permite avaliar o } \\
\text { grau de inconsistência da matriz de } \\
\text { julgamentos pariados (COSTA, 2006). }\end{array}$ \\
\hline 62 Passo & Eq. 6 & $\mathrm{RC}=\frac{\mathrm{IC}}{\mathrm{CA}}$ & $\begin{array}{l}\text { Razão de Consistência (RC). Permite avaliar a } \\
\text { inconsistência em função da ordem da matriz } \\
\text { de julgamentos. Caso o valor seja maior que } \\
0,10 \text { revisar o modelo e/ou os julgamentos } \\
\text { (COSTA, 2006). }\end{array}$ \\
\hline
\end{tabular}

Fonte: Mendes et. al. (2013)

Saaty (1980) argumenta que a grande vantagem do AHP é permitir aos seus usuários atribuir pesos relativos para múltiplos atributos, ou múltiplas alternativas para um dado atributo, ao mesmo tempo em que realiza uma comparação par a par entre os mesmos. Isso permite que, mesmo quando dois atributos são incompatíveis, a mente humana possa, ainda assim, reconhecer qual dos atributos é mais importante para o processo decisório.

Dessa forma, segundo Costa (2002) este método baseia-se em três etapas de pensamento analítico: construção de hierarquias, definição de prioridades, consistência lógica.

$\mathrm{Na}$ etapa de construção de hierarquias, o problema é estruturado em níveis hierárquicos descendentes formando uma hierarquia ou árvore de decisão no qual o objetivo geral deve ficar no topo da estrutura, sucedido dos critérios associados com o problema a ser solucionado. Tais critérios podem ser ainda diluídos em subcritérios, em um nível abaixo. Se houver necessidade, este processo poderá continuar até que todos os critérios sejam bem entendidos e especificados. Após os critérios, em um nível logo abaixo, são alocadas as alternativas.

Depois da construção da hierarquia, o decisor deve fazer uma comparação par a par, expressa em termos linguísticos/verbais, os quais serão convertidos em valores numéricos usando a Escala Fundamental de Saaty para julgamentos comparativos. Essa escala de valores, que varia entre 1 e 9, é capaz de expressar o grau de importância de uma alternativa em relação a cada critério e os critérios de um determinado nível em relação ao critério do nível imediatamente superior, como descrito na Tabela 1. 
Tabela 1: Escala fundamental de Saaty

\begin{tabular}{cll}
\hline $\mathbf{1}$ & \multicolumn{2}{c}{ ESCALA FUNDAMENTAL DE SAATY } \\
$\mathbf{3}$ & $\begin{array}{l}\text { Igual importância } \\
\text { Importância pequena de uma } \\
\text { sobre a outra }\end{array}$ & $\begin{array}{l}\text { As duas atividades contribuem igualmente para o objetivo. } \\
\text { A experiência e o juízo favorecem uma atividade em relação } \\
\text { a outra. }\end{array}$ \\
$\mathbf{5}$ & $\begin{array}{l}\text { Importância grande ou essencial } \\
\text { A experiência e o juízo favorece fortemente uma atividade } \\
\text { em relação a outra. }\end{array}$ \\
& $\begin{array}{l}\text { Importância muito grande ou } \\
\text { demonstrada }\end{array}$ & $\begin{array}{l}\text { Uma atividade é muito fortemente em relação a outra. Pode } \\
\text { ser demonstrada na prática. }\end{array}$ \\
$\mathbf{9}$ & Importância absoluta & $\begin{array}{l}\text { A evidencia favorece uma atividade em relação à outra, com } \\
\text { o mais alto grau de segurança. }\end{array}$ \\
& Quando se procura uma condição de compromisso entre duas \\
\end{tabular}

O Método TOPSIS foi originalmente apresentada por Hwang e Yoon em 1981. Segundo Lima Júnior e Carpinetti (2015), uma das vantagens do Método de Decisão Multicritério TOPSIS é a adequação aos problemas com grande número de critérios e alternativas, principalmente em casos onde os critérios são de natureza quantitativa.

Huang et al. (2011) e Samvedi et al. (2013), citam outras vantagens como: flexibilidade para ser combinado com análise estocástica; soluções obtidas de modo eficiente; procedimentos facilmente programados; resultados simples de serem compreendidos e aceitos pelos participantes do processo de decisão.

As etapas deste método se dão pela estrutura da Matriz de Decisão e o Vetor de Pesos, pois a partir desses valores será iniciado a aplicação dos cálculos. Na etapa seguinte é preciso normalizar a matriz de decisão utilizando a equação 1:

$$
n_{i j}=\frac{d_{i j}}{\sqrt{\sum_{j=1}^{n} d_{i j}^{2}}}
$$

Após a normalização, deve-se calcular a matriz de decisão ponderada e obter as soluções ideais positiva $(\mathrm{A}+)$ e negativa (A-) de acordo com as equações 2 a 6 :

$$
\begin{gathered}
n_{i j}=\frac{d_{i j}}{\sqrt{\sum_{j=1}^{n} d_{i j}^{2}}} \\
A^{+}=\left\{M A X_{j p_{i j}} \mid j=1,2, \cdots, m\right\}=\left\{n_{1}^{+}, \cdots, n_{j}^{+}, \cdots, n_{m}^{+}\right\} \\
A^{-}=\left\{M I N_{j p_{i j}} \mid j=1,2, \cdots, m\right\}=\left\{n_{1}^{-}, \cdots, n_{j}^{-}, \cdots, n_{m}^{-}\right\}
\end{gathered}
$$




$$
\begin{gathered}
D_{i}^{+}=\sqrt{\sum_{i=1}^{n}\left(n_{i j}-n_{j}^{+}\right)^{2}} \\
D_{i}^{-}=\sqrt{\sum_{i=1}^{n}\left(n_{i j}-n_{j}^{-}\right)^{2}}
\end{gathered}
$$

Para finalizar, deve-se calcular o coeficiente de aproximação $\left(\mathrm{CC}_{\mathrm{i}}\right)$ e quanto mais próximo de 1 for o valor, melhor será o desempenho da alternativa (equação 7).

$$
C C_{i}=\frac{D_{i}^{-}}{D_{i}^{+}+D_{i}^{-}}
$$

\section{Metodologia}

Com a finalidade de auxiliar na escolha do melhor meio de transporte para o trajeto entre Piabetá x Central do Brasil, foram levantadas informações para traçar o perfil da situação do Município de Magé. Em seguida foram definidos os autores relacionados ao processo, na prefeitura, diretamente com a secretaria de transporte, identificando-os de acordo com a classificação da metodologia multicritério de apoio à decisão.

Foi definida a metodologia aplicada pelas seguintes etapas: Definição dos critérios mais relevantes para a seleção do transporte, Coleta de Dados para a preparação do método e modelagem do problema, Aplicação dos cálculos para resolução do problema, utilizando priorização e ordenação através do software AHP TOPSIS $2 \mathrm{~N}$ e por fim, a análise dos resultados.

Figura 3 - Esquema metodológico utilizado

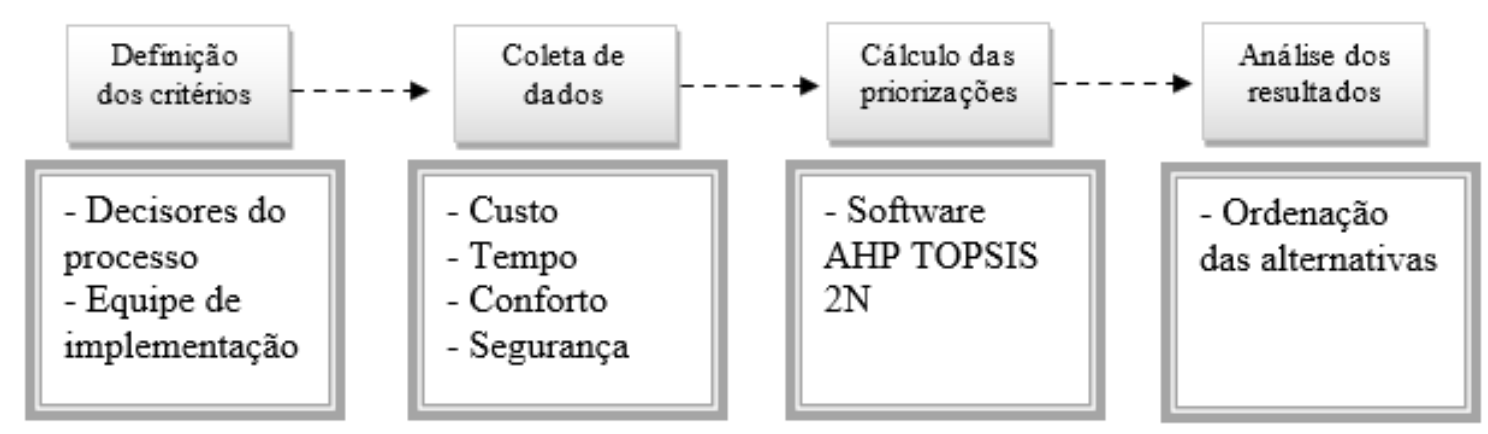

Fonte: Autores (2020)

Definidos os atores envolvidos no processo e as informações que permitiam uma melhor compreensão do contexto, foi possível avançar na identificação do melhor meio de transporte utilizado neste município do Rio de Janeiro.

Os meios de transporte em estudo foram escolhidos de acordo com a necessidade dos cidadãos. Sendo assim foi selecionado 4 meios de transporte: Ônibus, Carro (tendo em vista 
que os cidadãos compartilham o veículo com outras 4 pessoas para reduzir o custo da viagem), Van e Trem.

A próxima etapa se deu pela elaboração dos critérios e, em seguida, pela aplicação do modelo híbrido de AHP-TOPSIS-2N, para obtenção do resultado. A descrição dos critérios esta relacionada na Tabela 2:

Tabela 2: Descrição dos critérios

Fonte: Autores (2020)

Os graus foram parametrizados de "1" a " 5 ", sendo "1" o grau menos satisfatório sobre o critério do transporte e " 5 " o mais satisfatório, para a criação da Matriz de Decisão, constatada na Tabela 3.

Tabela 3: Matriz de Decisão

\begin{tabular}{ccccc}
\hline & CUSTO & TEMPO & SEGURANÇA & CONFORTO \\
\hline CARRO & $\mathrm{R} \$ 8,00$ & $2: 23 \mathrm{H}$ & 4 & 5 \\
ONIBUS & $\mathrm{R} \$ 8,00$ & $3: 35 \mathrm{H}$ & 2 & 3 \\
VAN & $\mathrm{R} \$ 10,00$ & $2: 40 \mathrm{H}$ & 1 & 2 \\
TREM & $\mathrm{R} \$ 4,20$ & $2: 07 \mathrm{H}$ & 3 & 1 \\
\hline
\end{tabular}

Fonte: Autores (2020)

Na sequência da definição da Matriz de Decisão, os critérios foram comparados par-a-par pelos decisores, que, juntos, aplicaram as notas, segundo a escala fundamental de Saaty. Após este procedimento, foi obtido a matriz importância com os pesos de cada critério, demonstrada na Tabela 4. Esta Matriz de Importância dos Critérios possui índice de consistência 0,0892 , menor que 0,10 , conforme recomendado pela literatura.

Tabela 4: Matriz de Importância dos Critérios

\begin{tabular}{ccccc}
\hline & CUSTO & TEMPO & SEGURANÇA & CONFORTO \\
\hline CUSTO & 1 & 3 & 5 & 7 \\
TEMPO & 0,33 & 1 & 5 & 7 \\
SEGURANÇA & 0,20 & 0,20 & 1 & 3 \\
CONFORTO & 0,14 & 0,14 & 0,33 & 1 \\
\hline
\end{tabular}

Fonte: Autores (2020)

\section{Resultados e Discussões}

\subsection{Descrição do Problema}

Na cidade do Rio de Janeiro, o impacto da ineficiência no deslocamento urbano é ainda maior. Para Pero e Mihessen (2012), a Região Metropolitana Rio de Janeiro (RMRJ) é onde as famílias destinam a maior parcela da renda com gastos em transporte. Mas os custos 
ocasionados por um sistema inadequado de transporte público vão muito além dos gastos diretos com passagens ou combustível: paralelo ao custo monetário das tarifas de transporte, há também os custos não monetizados (impacto na saúde e segurança do trabalhador, menos tempo disponível para crescimento intelectual/educacional, aumento do desemprego ocasionado pela perda de produtividade ou oportunidades de trabalho, aumento do uso de transportes ilegais e povoamento das periferias mais próximas dos centros, trocando qualidade de vida por proximidade do trabalho.

No caso do Estado do Rio de Janeiro, o público diretamente afetado é composto de trabalhadores que realizam longos deslocamentos para poder exercer sua ocupação. Deste grupo, moradores de regiões fora da capital e que se utilizam do transporte intermunicipal são os mais afetados, visto que optam por morar fora do centro, onde os custos de moradia são menores, e por outro lado sofrem a longa demora de cada jornada.

Segundo IPEA (2010), cerca de $15 \%$ do orçamento médio familiar brasileiro é destinado a arcar com custos de transporte. Este mesmo estudo mostra também que $32 \%$ das pessoas que fazem diariamente o percurso casa-trabalho gastam mais de uma hora por dia com o transporte. Os números são ainda piores quando se consideram apenas a população das regiões metropolitanas, onde $50 \%$ das pessoas gastam mais de uma hora por dia no trajeto. Entre as regiões metropolitanas estudadas, a RMRJ apresentou o pior resultado, com $56 \%$ da amostra declarando gastar mais de uma hora por dia no deslocamento casa - trabalho - casa.

O bairro mais populoso de Magé é Piabetá (cerca de 100.000 habitantes), e este dispõe de uma rodoviária com diversos destinos. Para o deslocamento dos moradores de Piabetá até a cidade do Rio de Janeiro, as alternativas são pelos modais rodoviários (ônibus, vans e veículo particular) ou ferroviários (trem).

Apesar do transporte ferroviário ser uma alternativa, o sistema atual é extremamente precário (estação sem infraestrutura adequada, trens sucateados e ausência de segurança pública), sendo uma alternativa que a maioria da população dispensa.

Utilizando o transporte público rodoviário, o morador de Magé que trabalha na Capital do Estado do Rio do Janeiro, com uma jornada de trabalho de 8 horas às 17 horas pega sua primeira condução às 5 horas, retornando para sua casa por volta das 19 horas, restando apenas como hora útil de descanso e lazer uma média de 4 horas.

\subsection{Modelagem matemática - Aplicação do método AHP-TOPSIS-2N}

Após a normalização da Matriz de Importância dos critérios, foi gerado o peso de cada critério em cada um dos meios de transporte estudados, como demonstrado na Tabela 5, utilizando o programa AHP TOPSIS $2 \mathrm{~N}$, desenvolvido por meio de uma parceria entre o Instituto Militar de Engenharia (IME), o Centro de Análises de Sistemas Navais (CASNAV) e a Universidade Federal Fluminense (UFF). 
Tabela 5: Matriz de Importância dos Critérios

\begin{tabular}{lr}
\hline \multicolumn{2}{c}{ PESOS } \\
\hline CUSTO & 0,5403 \\
TEMPO & 0,3119 \\
SEGURANÇA & 0,0993 \\
CONFORTO & 0,0485 \\
\hline \multicolumn{2}{c}{ Fonte: Autores $(2020)$}
\end{tabular}

Tabela 6: Consistência da Matriz de Ponderação dos Critérios

\begin{tabular}{lc}
\hline \multicolumn{2}{c}{ CONSISTÊNCIA } \\
\hline LAMBDA MÁX & 4,2586 \\
ÍNDICE DE CONSISTÊNCIA & 0,0862 \\
RAZÃO DE CONSISTÊNCIA & 0,0958 \\
\hline
\end{tabular}

Fonte: Autores (2020)

Com os pesos gerados de cada critério, foi possível normalizar e ponderar a matriz normalizada, gerando os resultados para o problema objeto de estudo.

\subsection{Classificação das Alternativas}

Ao final da aplicação do método AHP-TOPSIS-2N, chegou-se à classificação das alternativas, confome indica a Tabela 7.

Tabela 7: Classificação das alternativas

\begin{tabular}{lcc}
\hline & CLASSIFICAÇÃO & $\begin{array}{c}\text { PONTUAÇÃO DE } \\
\text { DESEMPENHO }\end{array}$ \\
\hline TREM & $1^{\text {o }}$ & 0,8800 \\
CARRO & $2^{\text {o }}$ & 0,4407 \\
ÔNIBUS & $3^{\text {o }}$ & 0,3141 \\
VAN & $4^{\text {o }}$ & 0,1980 \\
\hline
\end{tabular}

Fonte: Autores (2020)

Sendo assim, após apresentação dos resultados, chegou-se à conclusão de que o transporte público de melhor custo-benefício foi o trem. Em contrapartida, segundo dados coletados pela Supervia, de todas as outras alternativas, o trem é a menos utilizada. Isso evidencia a necessidade de iniciativa público-privadas para impulsionar o uso deste modal, através de melhorias estruturais para um melhor funcionamento daquele meio de transporte.

\section{Considerações finais}

O sistema de transporte exerce o papel de ligação entre pessoas e os espaços urbanos, destacando o grau de acessibilidade à população e o direito de ir e vir, garantido pela constituição brasileira. Também devem contribuir para a qualidade de vida, a ascensão e a promoção social dos cidadãos. Considerando o caráter social do transporte, sua disponibilidade deve ser garantida pelo estado, caracterizando-o como um serviço público.

No estado do Rio Janeiro, a ineficiência do transporte urbano impacta principalmente as famílias de renda mais baixa, sendo considerado um agravante social nesse aspecto. 
A pesquisa desenvolvida teve por objetivo, apresentar um estudo de caso real da aplicação da metodologia multicritério, no processo de tomada de decisão sobre a melhor alternativa de transporte dos moradores de Magé com destino ao Centro do Rio de Janeiro. Mediante aplicação do método AHP-TOPSIS-2N, chegou-se à conclusão de que o trem é a melhor alternativa a ser utilizada.

Como limitações deste estudo, destacam-se a falta de mensurar critérios subjetivos da percepção dos usuários sobre a qualidade de cada tipo de transporte, bem como fatores indiretos envolvidos em cada processo (manutenção dos carros particulares, superlotação do transporte público, nível de segurança de cada alternativa de transporte, dentre outros).

\section{REFERÊNCIAS}

BAGNASCHI, C. F.; Tomada de decisão em sistema de transporte urbano: uma análise multicritério. Manaus; 2012.

BELLA, R. L. F., Costa, H. G. Auxílio multicritério na priorização de riscos de projetos. Anais XLVIII Simpósio Brasileiro de Pesquisa Operacional. Vitória/ES, 2016.

CAITANO, A. L., Santos, M., Gomes, C. F. S., Tenório, F. M. Aplicação do Método Thor para escolha de um modelo de treinamento em um laboratório de metrologia. Anais XIX Simpósio de Pesquisa Operacional e Logísica da Marinha. Rio de Janeiro, 2019.

CARDOSO, C. Análise do transporte coletivo urbano sob a ótica dos riscos e carências sociais.Tese (Doutorado em Serviço Social), PUC, São Paulo, 2018.

COLENCI JR., A.; Kawamoto, E. Estimativa dos efeitos das condições de transporte na produtividade do trabalhador urbano. Relatório Final - DIRUR/Ipea. Brasília: IPEA, 1997.

Decision Support Systems Glossary. Disponível em: <http://dssresources.com/.> Acesso em jul. 2006.

DUARTE, F.; Sánchez, K.; Libardi, R. Introdução à mobilidade urbana. Curitiba: Juruá; 2012.

FIRJAN. FIRJAN. Disponível em: < https://www.firjan.com.br/ifdm/>. Acesso em: 18 nov. 2019.

GOMES, C.F.; Gomes, L.F.A.M. Tomada de decisão gerencial: Enfoque Multicritério. 4 ed. São Paulo: Atlas; 2012.

GRECO, S.; Ehrgott, M.; Figueira, J.F. Trends in Multiple Criteria decision analysis. New York: Springer, 2012. HUANG, I. B.; Keisler, J.; Linkov, I. Multi-criteria decision analysis in environmental sciences: Ten years of applications and trends. Science of the total environment, Elsevier, v. 409, n. 19, p. 3578-3594, 2011.

IPEA. A Nova Lei de Diretrizes da Política Nacional de Mobilidade Urbana. Comunicados do IPEA, No 128. Brasil. 2012. IPEA. A mobilidade urbana no Brasil. Comunicados do IPEA, Nº 94. Brasil, 2011. IPEA. Mobilidade urbana e posse de veículos: análise da PNAD 2009. Comunicados do IPEA, Nº 73. Brasil, 2014. JUNIOR, F. R. L.; Carpinetti, L. C. R. Uma comparação entre os métodos topsis e fuzzy-topsis no apoio à tomada de decisão multicritério para seleção de fornecedores. Gestão \& Produção, Directory of Open Access Journals, v. 22, n. 1, p. 17-34, 2015.

MENDONÇA, T. C., RANGEL, L. A. D. Priorização de equipamentos em manutenção empregando o apoio Multicritério à decisão. Anais XLIX Simpósio Brasileiro de Pesquisa Operacional. Blumenau/SC, 2017. MIRANDA, H.F.; Silva, A.N.R. Benchmarking sustainable urban mobility: The case of Curitiba, Brazil, Transport Policy, v. 21, p. $141-151 ; 2012$.

NTU. Associação Nacional Das Empresas De Transportes Urbanos. Anuário 18-19. Brasília; 2019.

ONU-Habitat. Estado de las Ciudades de América Latina y El Caribe 2012. Kenia. 2012.

SAATY, T. L., The Analytic Hierarchy Process: Planning, Priority, Setting and Resource Allocation, McGrawHill, Inc., 1980.

SAMVEDI, A.; Jain, V.; Chan, F. T. Quantifying risks in a supply chain through integration of fuzzy ahp and fuzzy topsis. International Journal of Production Research, Taylor \& Francis, v. 51, n. 8, p. 2433-2442, 2013. SANTOS, Marcos dos; Silva, A. M. T.; Lima, I. C.; Dias, F. C.; Martins, E. R. Application of AHP Method in the formation of a Performance Indicator for Operational Level Professionals. International Journal of Development Research, v. 06, issue 12, p. 10610-10615, 2016.

Scopus. Scopus Preview. Disponível em: <https://Www.scopus.com/home.uri>. Acesso em: 15 nov. 2019. SILVA, A. M. V. de A. da.; Melgarejo, L.; NOVAES, A. G. Análise de eficiência no transporte urbano por ônibus em municípios brasileiros. Artigo submetido ao XX Congresso da Associação Nacional de Pesquisa e Ensino em Transporte - ANPET, Anais de Congresso. Brasília-DF, 12p. 2006. Disponível em: Acesso em: 15 de Nov. de 2019. 
SOUZA, L.; Gomes, C. F. S.; Barros, A. Implementation of new Hybrid AHPTOPSIS-2N method in sorting and prioritizing of an it capex project portfolio. International Journal of Information Technology \& Decision Making. 10.1142/S0219622018500207; 2018.

TYLER, N. Accessibilty and the Bus System: Concepts to Practice. Institution of Civil Engineers - ICE. $1^{\text {a }}$ edição, Reino Unido, 2002.

VILAS BOAS, C. L. D. Análise da aplicação de métodos multicritérios de decisão na gestão de recursos hídricos. XVI SIMPÓSIO DE RECURSOS HÍDRICOS, Anais. João Pessoa-PB, 2005. 